[LV] O. Lehto and K. I. Virtanen, Quasiconformal Mappings in the Plane, 2nd ed., Grundlehren Math. Wiss. 126, Springer, New York, 1973.

P1] D. Partyka, Approximation of the Hersch-Pfluger distortion function. Applications, Ann. Univ. Mariae Curie-Skłodowska Sect. A 45 (1991), 99-111.

[P2] - Approximation of the Hersch-Pfiuger distortion function, Ann. Acad. Sci. Fenn. Ser. A I Math. 18 (1993), 343-354.

[P3] -, The maximal value of the function $[0,1] \ni r \mapsto \Phi_{K}^{2}(\sqrt{r})-r$, Bull. Soc. Sci. Lett. Łódź Sér. Rech. Déform. 20 (1995), 49-55.

[QVV] S.-L. Qiu, M. K. Vamanamurthy and M. Vuorinen, Bounds for quasiconformal distortion functions, J. Math. Anal. Appl., to appear.

[SC] A. Selberg and S. Chowla, On Epstein's zeta-function, J. Reine Angew. Math. 227 (1967), 87-110.

[S1] L.-C. Shen, On some cubic modular identities, Proc. Amer. Math. Soc. 110 (1993), 203-208.

[S2] -, On some modular equations of degree 5, ibid. 123 (1995), 1521-1526.

[VV] M. K. Vamanamurthy and M. Vuorinen, Functional inequalities, Jacobi products and quasiconformal maps, Mllinois J. Math. 38 (1994), 394-419.

Department of Mathematics

University of Helsinki

P.O. Box 4 (Yliopistonk. 5)

FIN-00014 Helsinki

Finland

E-mail: vuorinen@csc.fi

\section{Complex Unconditional Metric Approximation Property for $\mathcal{C}_{\Lambda}(\mathbb{T})$ spaces}

by

DANIEL LI (Paris)

Abstract. We study the Complex Unconditional Metric Approximation Property for translation invariant spaces $\mathcal{C}_{\Lambda}(\mathbb{T})$ of continuous functions on the circle group. We show that although some "tiny" (Sidon) sets do not have this property, there are "big" sets $\Lambda$ for which $\mathcal{C}_{\Lambda}(\mathbb{T})$ has $\left(\mathbb{C}\right.$-UMAP); though these sets are such that $L_{\Lambda}^{\infty}(\mathbb{T})$ contains functions which are not continuous, we show that there is a linear invariant lifting from these $L_{\Lambda}^{\infty}(\mathbb{T})$ which are not continuous, we show that

Introduction. The translation invariant subspaces of continuous functions on $\mathbb{T}$ all have the Metric Approximation Property (MAP). We study in this paper the spaces $\mathcal{C}_{\Lambda}(\mathbb{T})$ which satisfy a stronger approximation property, the Complex Unconditional Metric Approximation Property (C-UMAP).

The (Real) Unconditional Approximation Property (UMAP) was introduced in 1989 by P. Casazza and N. Kalton as an extreme possibility of approximation ([3], Th. 3.5), and they showed ([3], Th. 3.8) that it actually coincides for a separable Banach space $X$ with the existence for every $\varepsilon>0$ of an unconditional expansion of the identity of $X$ with constant $1+\varepsilon$, which means, by a result of A. Pelczyński and P. Wojtaszczyk ([21], Th. 1.1) that for every $\varepsilon>0, X$ may be isometrically embedded in a Banach space $Y$ with a $(1+\varepsilon)$-FDD for which there is a projection $P: Y \rightarrow X$ with $\|P\| \leq 1+\varepsilon$. Its complex version was defined and studied in ([7], $\S \S 8$ and 9 ).

To begin with, we construct subsets $\Lambda \subseteq \mathbb{Z}$ for which $\mathcal{C}_{\Lambda}(\mathbb{T})$ has (CUMAP). They are of two kinds: the first contain arbitrarily long arithmetical progressions, so that they are not $\Lambda(1)$-sets, but their pace tends to infinity; the second are Sidon sets, but have a pace which does not tend to infinity.

1991 Mathematics Subject Classification: Primary 46B20, 43A46, 41A65: Secondary $42 \mathrm{~A} 10,42 \mathrm{~A} 55,43 \mathrm{~A} 25$.

Key words and phrases: Unconditional Metric Approximation Property, translation invariant spaces of continuous functions, Rosenthal set, Riesz set, linear invariant lifting. 
Next, we show that the ( $\mathbb{C}$-UMAP) can always be achieved by convolution operators. This implies that if $\mathcal{C}_{\Lambda}$ has (C-UMAP), then so do $\mathcal{C}_{\Lambda_{0}}$ for all $\Lambda_{0} \subseteq \Lambda$, as well as all the spaces $L_{\Lambda}^{p}, 1 \leq p<\infty$.

When $\mathcal{C}_{\Lambda}$ has ( $\mathbb{C}$-UMAP), we remark that $\Lambda$ is a Rosenthal set if (and only if) $\mathcal{C}_{\Lambda}$ contains no subspace isomorphic to $c_{0}$. We show that this is not always the case: $\mathcal{C}_{\Lambda}$ can have ( $\mathbb{C}$-UMAP) when $\Lambda$ is a Hilbert set, and then $\mathcal{C}_{\Lambda}$ has subspaces isomorphic to $c_{0}$. However, we show that $\Lambda$ cannot contain any IP-set, nor the sum of two infinite sets. We also show that the uniform density of $\Lambda$ must be less than or equal to $1 / 2$, though it is likely that it is null. Finally, we show that for such a set there exists a linear invariant lifting from $L_{\Lambda}^{\infty}(\mathbb{T})$ into the Baire class 1 functions.

The notation is classical. $\mathbb{T}$ is the quotient $\mathbb{R} / 2 \pi \mathbb{Z}$ and for every $n \in \mathbb{Z}$ we denote by $e_{n}$ the character defined by $e_{n}(x)=e^{i n x}$ for $x \in \mathbb{T}$.

We recall that a complex (separable) Banach space $X$ has (C-UMAP) if there is a sequence of finite rank operators $R_{n}: X \rightarrow X$ such that

$$
\left\|R_{n} x-x\right\| \underset{n \rightarrow \infty}{\longrightarrow} 0 \text { for all } x \in X, \quad \sup _{|\lambda|=1}\left\|\mathrm{Id}-(1+\lambda) R_{n}\right\| \underset{n \rightarrow \infty}{\longrightarrow} 1 \text {. }
$$

When $\lambda$ belongs only to $\mathbb{R}$, instead of $\mathbb{C}, X$ is said to have (real) (UMAP).

I thank J. Chaumat, E. Fouvry, G. Godefroy, F. Lust-Piquard and G. Pisier for instructive conversations.

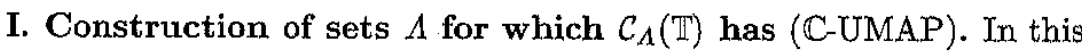
section, we give some examples of subsets $\Lambda \subset \mathbb{Z}$ for which $\mathcal{C}_{\Lambda}(\mathbb{T})$ has (C-UMAP).

It is worth mentioning that there is no Sidon subset $\Lambda \subseteq \mathbb{Z}$ with constant 1 whenever $\operatorname{card} \Lambda \geq 3$ ([2], p. 532).

LEMMA 1. For every finite subset $F \subseteq \mathbb{N}$ and every $\varepsilon>0$, there are $L$ intervals $I_{1}, \ldots, I_{L}$ of length $1 / L$ such that for each finite subset $G \subseteq \mathbb{N}$ there is an integer $n>\max F$ such that for every $f \in \mathcal{C}_{F}(\mathbb{T})$ and every $g \in \mathcal{C}_{G}(\mathbb{T})$, we have

$$
\left\|f+e_{n} g\right\|_{\mathcal{C}(\mathbb{T})} \geq \frac{1}{1+\varepsilon} \sup _{1 \leq l \leq L}\left(\|f\|_{\mathcal{C}\left(I_{l}\right)}+\|g\|_{\mathcal{C}\left(I_{l}\right)}\right)
$$

Proof. We may and do suppose that $\varepsilon \leq 3 / 4$.

Let $\pi_{F}: \mathcal{C}(\mathbb{T}) \rightarrow \mathcal{C}(\mathbb{T})$ be the projection which associates with $f \in \mathcal{C}(\mathbb{T})$ the trigonometric polynomial

$$
\pi_{F}(f)=\sum_{k \in F} \widehat{f}(k) e_{k} .
$$

Note that $\pi_{F}\left(f+e_{n} g\right)=f$ for every $f \in \mathcal{C}_{F}(\mathbb{T})$ and every $g \in \mathcal{C}_{G}(\mathbb{T})$ whenever $n>\max F$ and $G \subseteq \mathbb{N}$. Since $\mathcal{C}_{F}(\mathbb{T})$ is finite-dimensional, there exists a number $\varrho>0$ such that, for every $f \in \mathcal{C}_{F}(\mathbb{T})$, we have:

$$
|x-y| \leq \varrho \Rightarrow|f(x)-f(y)| \leq \frac{\varepsilon}{8\left\|\pi_{F}\right\|}\|f\|_{\infty} .
$$

We then divide $\mathbb{T}$ in $L$ intervals $I_{1}, \ldots, I_{L}$ of length $1 / L \leq \varrho$.

Sublemma. Let $M=(8 / \varepsilon)\left(1+\left\|\pi_{F}\right\|\right)$. Then there is an integer $n>$ $\max F$ such that for each $h \in M \cdot B_{\mathcal{C}_{G}(\mathbb{T})}$ and $1 \leq l \leq L$, we have

$$
\left\|1+e_{n} h\right\|_{\mathcal{C}\left(I_{l}\right)} \geq(1-\varepsilon / 2)\left(\|h\|_{\mathcal{C}\left(I_{l}\right)}+1\right) .
$$

Proof. Choose an $(\varepsilon / 8)$-net $h_{1}, \ldots, h_{K}$ of $M \cdot B_{\mathcal{C}_{G}(\mathbb{T})}$. We have the following:

ClaIM. Given $p$ non-void open intervals $L_{1}, \ldots, L_{p}$, there exist $y_{q} \in$ $L_{q}, 1 \leq q \leq p$, such that $1, y_{1}, \ldots, y_{p}$ are $\mathbb{Q}$-independent.

Indeed, if $1, t_{1}, \ldots, t_{p}$ are $\mathbb{Q}$-independent real numbers, we can choose $r_{q} \in\left(\frac{1}{t_{q}} L_{q}\right) \cap \mathbb{Q}^{*}, 1 \leq q \leq p$, and then the numbers $y_{q}=r_{q} t_{q}$ are suitable.

We can then choose $y_{1}^{l}, \ldots, y_{K}^{l} \in I_{l}$ such that

$$
\left|h_{k}\left(y_{k}^{l}\right)\right| \geq(1-\varepsilon / 8)\left\|h_{k}\right\|_{c\left(I_{l}\right)}
$$

and such that the set $\{1\} \cup\left\{y_{k}^{l}: 1 \leq l \leq L, 1 \leq k \leq K\right\}$ is $\mathbb{Q}$-independent. Then Kronecker's theorem ([10], Th. 442) enables us to find an integer $n>$ $\max F$ such that, for $1 \leq l \leq L$ and $1 \leq k \leq K$, we have

$$
\left|e^{i n y_{k}^{l}}-\frac{\left|h_{k}\left(y_{k}^{l}\right)\right|}{h_{k}\left(y_{k}^{l}\right)}\right| \leq \frac{\varepsilon}{8} \text {. }
$$

Then $\left|e^{i n y_{k}^{l}} h_{k}\left(y_{k}^{l}\right)-\right| h_{k}\left(y_{k}^{l}\right)|| \leq \frac{\varepsilon}{8}\left|h_{k}\left(y_{k}^{l}\right)\right|$, and so

$$
\begin{aligned}
\left\|1+e_{n} h_{k}\right\|_{\mathcal{C}\left(I_{l}\right)} & \geq\left|1+e^{i n y_{k}^{l}} h_{k}\left(y_{k}^{l}\right)\right| \\
& \geq 1+\left|h_{k}\left(y_{k}^{l}\right)\right|-\left|e^{i n y_{k}^{l}} h_{k}\left(y_{k}^{l}\right)-\right| h_{k}\left(y_{k}^{l}\right)|| \\
& \geq 1+\left|h_{k}\left(y_{k}^{l}\right)\right|-\frac{\varepsilon}{8}\left|h_{k}\left(y_{k}^{l}\right)\right| \geq 1+\left(1-\frac{\varepsilon}{8}\right)^{2}\left\|h_{k}\right\|_{\mathcal{C}\left(I_{l}\right)} .
\end{aligned}
$$

Now, for each $h \in M \cdot B_{\mathcal{C}_{G}(\mathbb{T})}$, there is an index $k$ such that

$$
\left\|h-h_{k}\right\|_{\mathcal{C}\left(I_{l}\right)} \leq\left\|h-h_{k}\right\|_{\mathcal{C}(\mathbb{T})} \leq \varepsilon / 8
$$

hence

$$
\begin{aligned}
\left\|1+e_{n} h\right\|_{\mathcal{C}\left(I_{l}\right)} & \geq\left\|1+e_{n} h_{k}\right\|_{\mathcal{C}\left(I_{l}\right)}-\left\|h-h_{k}\right\|_{\mathcal{C}_{\left(I_{l}\right)}} \\
& \geq(1-\varepsilon / 8)^{2}\left\|h_{k}\right\|_{\mathcal{C}\left(I_{l}\right)}+1-\varepsilon / 8 \\
& \geq(1-\varepsilon / 8)^{2}\left(\|h\|_{\mathcal{C}\left(I_{l}\right)}-\varepsilon / 8\right)+1-\varepsilon / 8 \\
& \geq(1-\varepsilon / 8)^{2}\|h\|_{\mathcal{C}\left(I_{l}\right)}+(1-\varepsilon / 8)^{3} \\
& \geq(1-\varepsilon / 2)\left(\|h\|_{\mathcal{C}\left(I_{l}\right)}+1\right)
\end{aligned}
$$


In the sequel we use the $n$ given by the sublemma. Let $f \in \mathcal{C}_{F}(\mathbb{T})$ and $g \in \mathcal{C}_{G}(\mathbb{T})$, and fix $l_{0} \in\{1, \ldots, L\}$ such that $\|f\|_{\mathcal{C}\left(I_{l_{0}}\right)}+\|g\|_{\mathcal{C}\left(I_{l_{0}}\right)}=$ $\sup _{1 \leq l \leq L}\left(\|f\|_{\mathcal{C}\left(I_{l}\right)}+\|g\|_{\mathcal{C}\left(I_{l}\right)}\right)$

We have two cases.

Assume first that $\|f\|_{C\left(I_{l_{0}}\right)} \leq(\varepsilon / 8)\left\|f+e_{n} g\right\|_{\mathcal{C}(\mathbb{T})}$. Then we observe first that since $l_{0}$ gives the maximum value, we have $\|f\|_{C\left(I_{l_{0}}\right)}+\|g\|_{\mathcal{C}\left(I_{l_{0}}\right)} \geq$ $\left\|f+e_{n} g\right\|_{\mathcal{C}(\mathbb{T})}$, and so

$$
\|g\|_{\mathcal{C}\left(I_{i_{0}}\right)} \geq\left(1-\frac{\varepsilon}{8}\right)\left\|f+e_{n} g\right\|_{\mathcal{C}(\mathbb{T})} \geq \frac{2+\varepsilon}{8}\left\|f+e_{n} g\right\|_{\mathcal{C}(\mathbb{T})}
$$

hence

$$
\begin{aligned}
\left\|f+e_{n} g\right\|_{\mathcal{C}(\mathbb{T})} & \geq\left\|f+e_{n} g\right\|_{\mathcal{C}\left(I_{l_{0}}\right)} \geq\|g\|_{\mathcal{C}\left(I_{l_{0}}\right)}-\|f\|_{\mathcal{C}\left(I_{I_{0}}\right)} \\
& \geq\|g\|_{\mathcal{C}\left(I_{l_{0}}\right)}-\frac{\varepsilon}{8}\left\|f+e_{n} g\right\|_{\mathcal{C}(\mathbb{T})} \\
& =\left(\frac{\|g\|_{\mathcal{C}\left(I_{l_{0}}\right)}}{\left\|f+e_{n} g\right\|_{\mathcal{C}(\mathbb{T})}}-\frac{\varepsilon}{8}\right)\left\|f+e_{n} g\right\|_{\mathcal{C}(\mathbb{T})} \\
& \geq \frac{1}{1+\varepsilon}\left(\frac{\|g\|_{\mathcal{C}\left(I_{l_{0}}\right)}}{\left\|f+e_{n} g\right\|_{\mathcal{C}(\mathbb{T})}}+\frac{\varepsilon}{8}\right)\left\|f+e_{n} g\right\|_{\mathcal{C}(\mathbb{T})} \\
& \text { since } \frac{\|g\|_{\mathcal{C}\left(I_{l_{0}}\right)}}{\left\|f+e_{n} g\right\|_{\mathcal{C}(\mathbb{T})}} \geq \frac{2+\varepsilon}{8} \\
& \geq \frac{1}{1+\varepsilon}\left(\|g\|_{\mathcal{C}\left(I_{l_{0}}\right)}+\frac{\varepsilon}{8}\left\|f+e_{n} g\right\|_{\mathcal{C}(\mathbb{T})}\right) \\
& \frac{1}{1+\varepsilon}\left(\|g\|_{\mathcal{C}\left(I_{l_{0}}\right)}+\|f\|_{\mathcal{C}\left(I_{l_{0}}\right)}\right) .
\end{aligned}
$$

Suppose now that $\|f\|_{C\left(I_{l_{0}}\right)} \geq(\varepsilon / 8)\left\|f+e_{n} g\right\|_{\mathcal{C}(\mathbb{T})}$. Let $x_{0} \in I_{l_{0}}$ be such that $\left|f\left(x_{0}\right)\right|=\|f\|_{\mathcal{C}\left(I_{l_{0}}\right)}$. For every $x \in I_{l_{0}}$, we have

$$
\begin{aligned}
\left\|f+e_{n} g\right\|_{\mathcal{C}(\mathbb{T})} & \geq\left|f\left(x_{0}\right)+e^{i n x} g(x)\right|-\left|f\left(x_{0}\right)-f(x)\right| \\
& \geq\|f\|_{\mathcal{C}\left(x_{i_{0}}\right)}\left|1+e^{i n x} \frac{g(x)}{f\left(x_{0}\right)}\right|-\frac{\varepsilon}{8\left\|\pi_{F}\right\|}\|f\|_{\mathcal{C}(\mathbb{T})} \\
& \geq\|f\|_{\mathcal{C}\left(I_{i_{0}}\right)}\left|1+e^{i n x} h(x)\right|-\frac{\varepsilon}{8}\left\|f+e_{n} g\right\|_{\mathcal{C}(\mathbb{T})}
\end{aligned}
$$

where $h=\frac{1}{f\left(x_{0}\right)} g \in(8 / \varepsilon)\left(1+\left\|\pi_{F}\right\|\right) \cdot B_{\mathcal{C}_{G}(\mathbb{T})}$, since

$$
\|g\|_{\infty}=\left\|e_{n} g\right\|_{\infty}=\left\|\left(\mathrm{Id}-\pi_{F}\right)\left(f+e_{n} g\right)\right\|_{\infty} \leq\left(1+\left\|\pi_{F}\right\|\right)\left\|f+e_{n} g\right\|_{\infty} .
$$

The previous inequalities can be read as

$$
\left\|f+e_{n} g\right\|_{\infty} \geq\|f\| \mathcal{C}\left(I_{l_{0}}\right)\left\|1+e_{n} h\right\|_{\mathcal{C}\left(I_{l_{0}}\right)}-\frac{\varepsilon}{8}\left\|f+e_{n} g\right\|_{\infty},
$$

and so the sublemma gives

$$
\begin{aligned}
\left\|f+e_{n} g\right\|_{\infty} & \geq \frac{1}{1+\varepsilon / 8}\|f\|_{\mathcal{C}\left(I_{l_{0}}\right)}\left\|1+e_{n} h\right\|_{\mathcal{C}\left(I_{l_{0}}\right)} \\
& \geq \frac{1}{1+\varepsilon / 8}\|f\|_{\mathcal{C}\left(I_{l_{0}}\right)}(1-\varepsilon / 2)\left(1+\|h\|_{\mathcal{C}\left(I_{l_{0}}\right)}\right) \\
& =\frac{1-\varepsilon / 2}{1+\varepsilon / 8}\left(\|f\|_{\mathcal{C}\left(I_{l_{0}}\right)}+\|g\|_{\mathcal{C}\left(I_{l_{0}}\right)}\right) \\
& \geq \frac{1}{1+\varepsilon}\left(\|f\|_{\mathcal{C}\left(I_{l_{0}}\right)}+\|g\|_{\mathcal{C}\left(I_{l_{0}}\right)}\right) \\
& =\frac{1}{1+\varepsilon} \sup _{1 \leq l \leq L}\left(\|f\|_{\mathcal{C}\left(I_{l}\right)}+\|g\|_{\mathcal{C}\left(I_{l}\right)}\right) .
\end{aligned}
$$

THEOREM 2. There exist subsets $\Lambda \subseteq \mathbb{Z}$ such that $\Lambda$ contains arbitrarily long arithmetical progressions and such that $\mathcal{C}_{\Lambda}(\mathbb{T})$ has (C-UMAP).

Remark. Then $\Lambda$ is not a $\Lambda(1)$-set ([25], Th. 4.1), that is to say, $L_{\Lambda}^{1}$ is not reflexive (by [13] and [1]; see also [22]).

In order to prove this theorem, we need the following lemma.

LEMMA 3. For every $\varepsilon>0$ and every finite set $F \subseteq \mathbb{N}$, there is an $L \geq 1$ such that for every finite set $G \subseteq L \mathbb{N}^{*}$, there is $n>\max F$ such that

$$
\left\|f+e_{n} g\right\|_{\infty} \geq \frac{1}{1+\varepsilon}\left(\|f\|_{\infty}+\|g\|_{\infty}\right)
$$

for every $f \in \mathcal{C}_{F}$ and every $g \in \mathcal{C}_{G}$.

Proof. This follows from Lemma 1 , since $G \subseteq L \mathbb{N}^{*}$ implies that every $g \in \mathcal{C}_{G}(\mathbb{T})$ has period $1 / L$ and so $\|g\|_{\infty}=\|g\|_{\mathcal{C}\left(I_{l}\right)}$ for every $l \in\{1, \ldots, L\}$; in particular, Lemma 3 is obtained by considering $l_{0}$ such that $\|f\|_{\infty}=$ $\|f\|_{C\left(I_{l_{0}}\right)}$.

Proof of $\mathrm{T}$ heorem 2 . The construction of $\Lambda$ will be done by induction. First, we apply Lemma 3 with $\varepsilon=\varepsilon_{1}$ and $F=\{0\}$; we find a suitable $L_{1} \geq 1$ and with $G=\left\{L_{1}\right\}$, we now find an $n_{1} \geq 1$; we set $\Lambda_{1}=\left\{0, L_{1}+n_{1}\right\}$.

Suppose now that we have constructed a set $\Lambda_{k}=H_{0} \cup \ldots \cup H_{k} \subseteq \mathbb{N}$ where $H_{0}=\{0\}$ and $H_{j}=n_{j}+\left\{L_{j}, 2 L_{j}, \ldots, j L_{j}\right\}, 1 \leq j \leq k$, are disjoint sets such that for $1 \leq j \leq k$,

$$
\left\|\sum_{l=0}^{k} f_{l}\right\|_{\infty} \geq \frac{1}{\left(1+\varepsilon_{j}\right) \ldots\left(1+\varepsilon_{k}\right)}\left(\left\|\sum_{l=0}^{j-1} f_{l}\right\|_{\infty}+\sum_{l=j}^{k}\left\|f_{l}\right\|_{\infty}\right)
$$

where $f_{l} \in \mathcal{C}_{H_{l}}, 0 \leq l \leq k$, and where $\varepsilon_{1}, \ldots, \varepsilon_{k}>0$ have been chosen in 
such a way that

$$
\prod_{l=j}^{k}\left(1+\varepsilon_{l}\right) \leq 1+\left(1+\ldots+\frac{1}{2^{k-j-1}}\right) \varepsilon_{j}, \quad 1 \leq j \leq k-1 .
$$

We apply Lemma 3 again with $\varepsilon=\varepsilon_{k+1}$ chosen such that

$$
\prod_{l=j}^{k+1}\left(1+\varepsilon_{l}\right) \leq 1+\left(1+\ldots+\frac{1}{2^{k-j}}\right) \varepsilon_{j}, \quad 1 \leq j \leq k,
$$

and with $F=\Lambda_{k} ;$ we find $L_{k+1}$ and, taking

$$
G=G_{k+1}=\left\{L_{k+1}, 2 L_{k+1}, \ldots,(k+1) L_{k+1}\right\},
$$

we obtain $n_{k+1}>\max \Lambda_{k}$. We then set

$$
\Lambda_{k+1}=\Lambda_{k} \cup\left(n_{k+1}+G_{k+1}\right) .
$$

The formulas (1) and (2) show that

$$
\sup _{|\lambda|=1}\left\|\operatorname{Id}-(1+\lambda) \pi_{\Lambda_{j}}\right\|_{\mathcal{L}\left(\mathcal{C}_{A_{k+1}}\right)} \leq 1+\left(1+\ldots+\frac{1}{2^{k-j}}\right) \varepsilon_{j}
$$

Now $\Lambda=\bigcup_{k \geq 1} \Lambda_{k}$ is a set such that $\mathcal{C}_{\Lambda}(\mathbb{T})$ has (C-UMAP) since

$$
\sup _{|\lambda|=1}\left\|\operatorname{Id}-(1+\lambda) \pi_{\Lambda_{j}}\right\|_{\mathcal{L}\left(\mathcal{C}_{\Lambda}\right)} \leq 1+2 \varepsilon_{j} .
$$

Remark. This construction, though different, follows the same idea as H. P. Rosenthal's ([24]). We obtain a Rosenthal set of " $\ell_{1}$-sum" type.

THEOREM 4. For every finite set $F_{0} \subseteq \mathbb{N}$, there is an increasing sequence $\left(n_{k}\right)_{k \geq 0}$ of integers such that $\mathcal{C}_{\Lambda}(\mathbb{T})$ has (C-UMAP) with $\Lambda=\bigcup_{k \geq 0}\left(n_{k}+F_{0}\right)$. In particular, the pace of $\Lambda$ does not tend to infinity.

Proof. We first remark that for every $u, v \in \mathcal{C}(\mathbb{T})$, we have

$$
\sup _{|\lambda|=1}\|u+\lambda v\|_{\infty}=\sup _{x \in \mathbb{T}}(|u(x)|+|v(x)|) \text {. }
$$

Starting from $n_{0}=0$, we apply Lemma 1 with $F=G=F_{0}$. We obtain an integer $n_{1}>\max F_{0}=N_{0}$ such that for $f, g \in \mathcal{C}_{F_{0}}(\mathbb{T})$ and $|\lambda|=1$, we have

$$
\left\|-\lambda f+e_{n_{1}} g\right\|_{\infty} \leq\left(1+\varepsilon_{1}\right)\left\|f+e_{n_{1}} g\right\|_{\infty} .
$$

Hence, on setting $\Lambda_{0}=F_{0}$ and $\Lambda_{1}=\Lambda_{0} \cup\left(n_{1}+\Lambda_{0}\right)$, we have

$$
\sup _{|\lambda|=1}\left\|\operatorname{Id}-(1+\lambda) \pi_{\Lambda_{0}}\right\|_{\mathcal{L}\left(C_{\Lambda_{1}}\right)} \leq 1+\varepsilon_{1}
$$

Suppose now we have already constructed integers $n_{1}, \ldots, n_{k}$ such that

$$
N_{0}+n_{j-1}<n_{j}, \quad 1 \leq j \leq k,
$$

and $\varepsilon_{1}>\ldots>\varepsilon_{k}>0$ such that

$$
\prod_{l=j}^{k}\left(1+\varepsilon_{l}\right) \leq 1+\left(1+\ldots+\frac{1}{2^{k-j-1}}\right) \varepsilon_{j}
$$

and such that, for $1 \leq j \leq k$, we have

$$
\sup _{|\lambda|=1}\left\|\operatorname{Id}-(1+\lambda) \pi_{\Lambda_{j-1}}\right\|_{\mathcal{L}\left(\mathcal{C}_{\Lambda_{k}}\right)} \leq\left(1+\varepsilon_{j}\right) \ldots\left(1+\varepsilon_{k}\right),
$$

where we have set $\Lambda_{k}=\bigcup_{j=0}^{k}\left(n_{j}+F_{0}\right)$.

We now apply Lemma 1 with $F=\Lambda_{k}$ and $G=F_{0}$, and $\varepsilon=\varepsilon_{k+1}>0$ chosen in such a way that $\varepsilon_{k+1}<\varepsilon_{k}$ and such that for $1 \leq j \leq k$,

$$
\left(1+\varepsilon_{j}+\frac{\varepsilon_{j}}{2}+\ldots+\frac{\varepsilon_{j}}{2^{k-j-1}}\right)\left(1+\varepsilon_{k+1}\right) \leq 1+\varepsilon_{j}+\frac{\varepsilon_{j}}{2}+\ldots+\frac{\varepsilon_{j}}{2^{k-j-1}}+\frac{\varepsilon_{j}}{2^{k-j}} \text {. }
$$

We then find an integer $n_{k+1}>\max \Lambda_{k}=N_{0}+n_{k}$ and $L$ intervals $I_{1}, \ldots, I_{L}$ of length $1 / L$ such that

$$
\|(u+v)+w\|_{\infty} \geq \frac{1}{1+\varepsilon_{k+1}} \sup _{1 \leq l \leq L}\left(\|u+v\|_{\mathcal{C}\left(I_{l}\right)}+\|w\|_{\mathcal{C}\left(I_{l}\right)}\right)
$$

for $u \in \mathcal{C}_{\Lambda_{j}}, v \in \mathcal{C}_{\Lambda_{k} \backslash \Lambda_{j}}$ and $w \in \mathcal{C}_{n_{k+1}+F_{0}}$

In particular, we have

$$
\sup _{|\lambda|=1}\left\|\operatorname{Id}-(1+\lambda) \pi_{A_{k}}\right\|_{\mathcal{L}\left(\mathcal{C}_{A_{k+1}}\right)} \leq\left(1+\varepsilon_{j}\right) \ldots\left(1+\varepsilon_{k+1}\right) .
$$

Writing now $w=e_{n_{k+1}} u_{1}$ with $u_{1} \in \mathcal{C}_{F_{0}}(\mathbb{T})$, we have

$$
\begin{aligned}
\|(u+v)+ & w \|_{\infty} \geq \frac{1}{1+\varepsilon_{k+1}} \sup _{x \in \mathbb{T}}\left(|u(x)+v(x)|+\left|u_{1}(x)\right|\right) \\
& \geq \frac{1}{1+\varepsilon_{k+1}} \sup _{x \in \mathbb{T}} \sup _{|\sigma|=1}\left(\left|u(x)+\sigma u_{1}(x)+v(x)\right|\right) \\
& =\frac{1}{1+\varepsilon_{k+1}} \sup _{|\sigma|=1}\left\|\left(u+\sigma u_{1}\right)+v\right\|_{\infty} \\
& \geq \frac{1}{1+\varepsilon_{k+1}} \cdot \frac{1}{\left(1+\varepsilon_{j}\right) \ldots\left(1+\varepsilon_{k}\right)} \sup _{|\sigma|=1} \sup _{|\lambda|=1}\left\|-\lambda\left(u+\sigma u_{1}\right)+v\right\|_{\infty} \\
& =\frac{1}{\left(1+\varepsilon_{j}\right) \ldots\left(1+\varepsilon_{k}\right)\left(1+\varepsilon_{k+1}\right)} \sup _{x \in \mathbb{T}}\left(|u(x)|+\left|u_{1}(x)\right|+|v(x)|\right) \\
& \geq \frac{1}{\left(1+\varepsilon_{j}\right) \ldots\left(1+\varepsilon_{k+1}\right)} \sup _{x \in \mathbb{T}}\left(|u(x)|+\left|v(x)+e^{i n_{k+1} x} u_{1}(x)\right|\right) \\
& \geq \frac{1}{\left(1+\varepsilon_{j}\right) \ldots\left(1+\varepsilon_{k+1}\right)} \sup _{|\lambda|=1}\|-\lambda u+(v+w)\|_{\infty} ;
\end{aligned}
$$


hence

$$
\sup _{|\lambda|=1}\left\|\operatorname{Id}-(1+\lambda) \pi_{A_{j}}\right\|_{\mathcal{L}\left(\mathcal{C}_{A_{k+1}}\right)} \leq\left(1+\varepsilon_{j}\right) \ldots\left(1+\varepsilon_{k+1}\right) .
$$

Setting now

$$
\Lambda=\bigcup_{k \geq 0} \Lambda_{k}=\bigcup_{k \geq 0}\left(n_{k}+F_{0}\right)
$$

we obviously have for all $j \geq 0$,

$$
\sup _{|\lambda|=1}\left\|\operatorname{Id}-(1+\lambda) \pi_{\Lambda_{j}}\right\|_{\mathcal{L}\left(C_{A}\right)} \leq 1+2 \varepsilon_{j} .
$$

Since $\lim _{j \rightarrow \infty} \varepsilon_{j}=0$, the space $\mathcal{C}_{\Lambda}(\mathbb{T})$ does have (C-UMAP).

Remark. The proof shows that $\left\{n_{k}\right\}_{k \geq 1}$ is a Sidon set with constant $1+2 \varepsilon_{1}$. Since $F_{0}$ is a finite set, Drury's theorem implies that the above constructed set $\Lambda$ is itself a Sidon set, as a finite union of Sidon sets.

II. Properties implied by (C-UMAP) for $\mathcal{C}_{\Lambda}(\mathbb{T})$ spaces. Most of the properties below do not depend on the particular nature of the group $\mathbb{T}$, so we state them in the abstract setting of a compact metrizable abelian group $G$.

First, we are going to see that the approximation can be achieved with convolution operators.

LEMMA 5. If $\mathcal{C}_{\Lambda}(G)$ has (C-UMAP), there exists a sequence of finite rank convolution operators $C_{n}: \mathcal{C}_{\Lambda}(G) \rightarrow \mathcal{C}_{\Lambda}(G)$ such that

$$
\left\|f-C_{n} f\right\|_{\infty} \underset{n \rightarrow \infty}{\longrightarrow} 0 \text { and } \sup _{|\lambda|=1}\left\|\operatorname{Id}-(1+\lambda) C_{n}\right\| \underset{n \rightarrow \infty}{\longrightarrow} 1 \text {. }
$$

Proof. Let $R_{n}: \mathcal{C}_{\Lambda} \rightarrow \mathcal{C}_{\Lambda}$ be finite rank operators such that $\| R_{n} f$ $f \|_{\infty} \rightarrow 0$ for every $f \in \mathcal{C}_{\Lambda}(G)$ and $\sup _{|\lambda|=1}\left\|\mathrm{Id}-(1+\lambda) R_{n}\right\| \rightarrow 1$ as $n \rightarrow \infty$.

Let $\left(K_{l}\right)_{l \geq 1}$ be an approximate unit, and $S_{l}: \mathcal{C}_{\Lambda} \rightarrow \mathcal{C}_{\Lambda}$ be the convolution operator associated with $K_{l}$. Since $R_{n}$ has finite rank, by using a $2^{-(n+2)}$-net in $R_{n}\left(B_{\mathcal{C}_{A}}\right)$, we can find an index $l_{n}$ such that

$$
\left\|R_{n}-S_{l_{n}} R_{n}\right\| \leq 2^{-n}
$$

The operator $T_{n}=S_{l_{n}} R_{n}$, satisfies

$$
\left\|f-T_{n} f\right\|_{\infty} \leq\left\|f-R_{n} f\right\|_{\infty}+\left\|R_{n} f-S_{l_{n}} R_{n} f\right\|_{\infty} \underset{n \rightarrow \infty}{\longrightarrow} 0
$$

and

$$
\begin{aligned}
\left\|\operatorname{Id}-(1+\lambda) T_{n}\right\| & \leq\left\|\mathrm{Id}-(1+\lambda) R_{n}\right\|+2\left\|R_{n}-S_{l_{n}} R_{n}\right\| \\
& \leq\left\|\mathrm{Id}-(1+\lambda) R_{n}\right\|+2^{-n+1}
\end{aligned}
$$

so that $\sup _{|\lambda|=1}\left\|\operatorname{Id}-(1+\lambda) T_{n}\right\| \rightarrow 1$ as $n \rightarrow \infty$. Moreover, $T_{n}\left(\mathcal{C}_{\Lambda}\right) \subseteq \mathcal{P}_{\Lambda}$ (where $\mathcal{P}_{\Lambda}$ is the set of trigonometric polynomials with spectrum in $\Lambda$ ).
Now, set

$$
\widetilde{T}_{n}(f)=\int_{G}\left[T_{n}\left(f_{x}\right)\right]_{-x} d x .
$$

Since $T_{n}\left(\mathcal{C}_{\Lambda}\right)$ is finite-dimensional and $T_{n}\left(\mathcal{C}_{\Lambda}\right) \subseteq \mathcal{P}_{\Lambda}$, there is a finite collection of characters $\Lambda_{n}=\left\{\gamma_{1}, \ldots, \gamma_{N}\right\}$ such that $T_{n}\left(\mathcal{C}_{\Lambda}\right) \subseteq \mathcal{P}_{\Lambda_{n}}$, so that $\widetilde{T}_{n}\left(\mathcal{C}_{\Lambda}\right) \subseteq \mathcal{P}_{\Lambda_{n}}$ is also finite-dimensional. Moreover, $\left\|\widetilde{T}_{n} f-f\right\|_{\infty} \rightarrow 0$ for each $f \in \mathcal{C}_{\Lambda}$, and $\sup _{|\lambda|=1}\left\|\mathrm{Id}-(1+\lambda) \widetilde{T}_{n}\right\| \rightarrow 1$ as $n \rightarrow \infty$. A direct computation gives that $C_{n}=\widetilde{T}_{n}$ is the convolution operator associated with the trigonometric polynomial $P_{n}=\sum_{\gamma \in \Lambda_{n}} \widehat{T_{n}(\gamma)}(\gamma) \gamma$.

Corollary 6. If $\mathcal{C}_{\Lambda}(G)$ has (C-UMAP), then so does $\mathcal{C}_{\Lambda_{0}}(G)$ for every $\Lambda_{0} \subseteq \Lambda$.

We now give some consequences of this result.

THEOREM 7. If $\mathcal{C}_{\Lambda}(G)$ has (C-UMAP), then so do all the spaces $L_{\Lambda}^{\mathcal{P}}(G)$, $1 \leq p<\infty$.

Proof. That follows from Lemma 5 and from the following observation. Let $P$ be a trigonometric polynomial, and $C$ be the associated convolution operator. Then we have

$$
\left(\forall g \in \mathcal{P}_{\Lambda}\right) \quad\|g-P * g\|_{p} \leq\|g-P * g\|_{\infty},
$$

and

$$
\|\mathrm{Id}-(1+\lambda) C\|_{\mathcal{L}\left(L_{\Lambda}^{p}\right)} \leq\left\|\delta_{0}-(1+\lambda) P\right\|_{\mathcal{M} / \mathcal{M}_{A^{c}}}=\|\operatorname{Id}-(1+\lambda) C\|_{\mathcal{L}\left(\mathcal{C}_{\Lambda}\right)}
$$

(see [11], Th. 2)

Remark. By ([6], Prop. 2.4) and ([8], Th. II.2), if $L_{\Lambda}^{1}$ has (C-UMAP) and so (UMAP), then $\Lambda$ is a Shapiro set ([4], Def. 1.6); in particular, it is a Riesz set ([4], Th. 1.9). Moreover, it then follows from ([7], Th. 9.2) that the predual $\mathcal{C} / \mathcal{C}_{\Lambda^{\prime}}$ of $L_{\Lambda}^{1}$ also has ( $\mathbb{C}$-UMAP), and more precisely, there is a sequence of finite rank operators $A_{n}: \mathcal{C} / \mathcal{C}_{\Lambda^{\prime}} \rightarrow \mathcal{C} / \mathcal{C}_{\Lambda^{\prime}}$ such that

$$
\begin{aligned}
& \left\|A_{n} \varphi-\varphi\right\| \underset{n \rightarrow \infty}{\longrightarrow} 0, \quad \forall \varphi \in \mathcal{C} / \mathcal{C}_{\Lambda^{\prime}}, \\
& \left\|A_{n}^{*} f-f\right\|_{1} \underset{n \rightarrow \infty}{\longrightarrow} 0, \quad \forall f \in L_{\Lambda}^{1}, \\
& \sup _{|\lambda|=1}\left\|\operatorname{Id}-(1+\lambda) A_{n}\right\|_{n \rightarrow \infty} 1 .
\end{aligned}
$$

Now, given an approximate unit $\left(K_{n}\right)_{n \geq 1}$, by ([14], Lemma 1) we have $A_{n}$ $K_{n} \rightarrow 0$ weakly in $\mathcal{K}\left(\mathcal{C} / \mathcal{C}_{\Lambda^{\prime}}\right)$ as $n \rightarrow \infty$ so that there are finite convex blocks $C_{n}$ of the $K_{n}$ 's such that the above three conditions are also true with $C_{n}$ instead of $A_{n}$. That gives: 
Proposition 8. Let $G$ be an abelian compact metrizable group and $T$ its

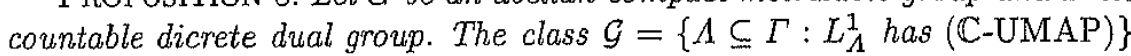
is an $F_{\sigma \delta}$ in $\mathcal{P}(\Gamma)$.

The topology on $\mathcal{P}(\Gamma)$ is the product topology of $\{0,1\}^{\Gamma}$, where $\mathcal{P}(\Gamma)$ and $\{0,1\}^{\Gamma}$ are identified by the map $\Lambda \mapsto \mathbf{1}_{\Lambda}$.

Proof. Let $\left(K_{n}\right)_{n \geq 1}$ be a given approximate unit, and $\mathcal{B}$ the set of all the rational finite convex blocks of the $K_{n}$ 's; it is a countable set, and we have

$$
\mathcal{G}=\bigcap_{n \geq 1} \bigcup_{R \in \mathcal{B}} \mathcal{G}_{n, R}
$$

where

$$
\mathcal{G}_{n, R}=\left\{\Lambda \subseteq \Gamma: \sup _{|\lambda|=1}\|\operatorname{Id}-(1+\lambda) R\|_{\mathcal{L}\left(L_{\Lambda}^{1}\right)} \leq 1+1 / n\right\}
$$

We thus obtain the proposition since this last set is closed in $\mathcal{P}(\Gamma)$ : if $\Lambda_{j} \rightarrow \Lambda$ in $\mathcal{P}(\Gamma)$ as $n \rightarrow \infty$, with $\Lambda_{j} \in \mathcal{G}_{n, R}$, let $P \in \mathcal{P}_{\Lambda}$; since $\operatorname{spec}(P) \subseteq \Lambda$ is a finite set, there is an index $j$ such that $\operatorname{spec}(P) \subseteq \Lambda_{j}$, and so $\sup _{|\lambda|=1} \| P$ $(1+\lambda) R * P\left\|_{1} \leq(1+1 / n)\right\| P \|_{1}$.

It is proved in ([6], Prop. 2.8) that if a Banach space with (UMAP) does not contain any subspace isomorphic to $c_{0}$, then it is isometric to a (separable) dual Banach space; in particular, such a space has the RadonNikodym Property. From the characterization of F. Lust-Piquard ([16]), we obtain:

Proposition 9. If $\mathcal{C}_{\Lambda}(G)$ has (UMAP) and contains no subspace isomorphic to $c_{0}$, then $\Lambda$ is a Rosenthal set.

Let us recall that a Rosenthal set is a set $\Lambda$ for which $\mathcal{C}_{\Lambda}=L_{\Lambda}^{\infty}$ and that no example is known of a non-Rosenthal set $\Lambda$ for which $\mathcal{C}_{\Lambda}$ does not contain any subspace isomorphic to $c_{0}$.

However, there are non-Rosenthal sets $A$ for which $\mathcal{C}_{\Lambda}(\mathrm{T})$ has (C-UMAP):

THEOREM 10. There are Hilbert sets $\Lambda \subseteq \mathbb{Z}$ for which $\mathcal{C}_{\Lambda}(\mathbb{T})$ has (C-UMAP).

Let us recall that a Hilbert set $\Lambda$ is defined by

$$
\Lambda=\bigcup_{n \geq 1}\left\{q_{n}+\sum_{k=1}^{n} \varepsilon_{k} p_{k}: \varepsilon_{k}=0 \text { or } 1\right\}
$$

where $\left(q_{n}\right)_{n \geq 1}$ and $\left(p_{n}\right)_{n \geq 1}$ are two sequences in $\mathbb{Z}, p_{n} \neq 0$, and that $\mathcal{C}_{\Lambda}(\mathbb{T})$ has subspaces isomorphic to $c_{0}$ for any Hilbert set $\Lambda$ ([15], Th. 2); in particular, Hilbert sets are never Rosenthal sets.

Proof of Theorem 10. It is a consequence of the following
Theorem (Y. Meyer ([19], Ch. VIII, § 5.1, Th. IV, p. 247). Let $\left(t_{k}\right)_{k \geq 1}$ be an increasing sequence of positive numbers such that

(a) $t_{k+1}>s_{k}=t_{1}+\ldots+t_{k}$,

(b) $\sum_{k=1}^{\infty}\left(t_{k} / t_{k+1}\right)^{2}<\infty$

Then the IP-set

$$
\Lambda_{\infty}=\left\{\sum_{k=1}^{n} \varepsilon_{k} t_{k}: \varepsilon_{k}=0 \text { or } 1, n \geq 1\right\}
$$

has the following property: for every $\varepsilon>0$, there is an integer $m_{0} \in \mathbb{N}$ for which

$$
\left\|P_{m_{0}}\right\|_{\infty} \leq(1+\varepsilon)\|P\|_{\infty}
$$

where $P$ is any trigonometric polynomial with spectrum in $\Lambda_{\infty}$ :

$$
P(x)=\sum_{\varepsilon_{k}=0,1} a_{\left(\varepsilon_{1}, \ldots, \varepsilon_{n}\right)} e^{2 \pi i\left(\sum_{k=1}^{n} \varepsilon_{k} t_{k}\right) x}
$$

and

$$
P_{m}\left(x, x_{m+1}, \ldots, x_{n}\right)=\sum_{\varepsilon_{k}=0,1} a_{\left(\varepsilon_{1}, \ldots, \varepsilon_{n}\right)} e^{2 \pi i\left[\left(\sum_{k=1}^{m} \varepsilon_{k} t_{k}\right) x+\sum_{k=m+1}^{n} \varepsilon_{k} x_{k}\right]} .
$$

It is then clear that, for integers $t_{k}$, the Hilbert set $\Lambda \subseteq \Lambda_{\infty}$ defined by

$$
\Lambda=\bigcup_{n \geq 1}\left\{t_{2 n}+\sum_{k=1}^{n} \varepsilon_{k} t_{2 k-1}: \varepsilon_{k}=0 \text { or } 1\right\}
$$

satisfies

$$
\sup _{x \in \mathbb{T}}\left[\left|\left(\pi_{\Lambda_{m}} P\right)(x)\right|+\mid P(x)-\left(\pi_{\Lambda_{m}} P\right)(x)\|\leq\| P_{2 m}\left\|_{\infty} \leq(1+\varepsilon)\right\| P \|_{\infty}\right.
$$

for every $m \geq m_{0}$, where

$$
\Lambda_{m}=\bigcup_{n=1}^{m}\left\{t_{2 n}+\sum_{k=1}^{n} \varepsilon_{k} t_{2 k-1}: \varepsilon_{k}=0 \text { or } 1\right\}
$$

and that means that for $m \geq m_{0}$,

$$
\sup _{|\lambda|=1}\left\|\operatorname{Id}-(1+\lambda) \pi_{\Lambda_{m}}\right\| \leq 1+\varepsilon
$$

and so $\mathcal{C}_{\Lambda}(\mathbb{T})$ has $(\mathbb{C}-\mathrm{UMAP})$.

Indeed, we can first see that assumption (a) implies that the sets $\Lambda_{m+1} \backslash$ $\Lambda_{m}$ are disjoint and that we can write

$$
P(x)=\left(\pi_{\Lambda_{m}} P\right)(x)+\sum_{k=1}^{[n / 2]-m+1} e^{2 \pi i t_{2 m+2 k} x} Q_{k}(x)
$$


where $Q_{k}$ are trigonometric polynomials with spectrum in $\left\{\sum_{j=1}^{m+k} \varepsilon_{j} t_{2 j-1}\right.$ : $\varepsilon_{j}=0$ or 1$\}$; therefore

$$
\begin{aligned}
& P_{2 m}\left(x, x_{2 m+1}, x_{2 m+2}, \ldots, x_{n}\right) \\
& =\left(\pi_{\Lambda_{m}} P\right)(x)+\sum_{k=1}^{[n / 2]-m+1} e^{2 \pi i x_{2 m+2 k}} Q_{k}\left(x, x_{2 m+1}, x_{2 m+3}, \ldots, x_{2 m+2 k-1}\right),
\end{aligned}
$$

so, taking the supremum over $x_{2 m+2}, x_{2 m+4}, \ldots, x_{2[n / 2]+2}$, and setting $x_{2 m+1}=x_{2 m+3}=\ldots=x$, we get

$$
\begin{aligned}
\left\|P_{2 m}\right\|_{\infty} & \geq \sup _{x \in \mathbb{T}}\left[\left|\left(\pi_{\Lambda_{m}} P\right)(x)\right|+\sum_{k=1}^{[n / 2]-m+1}\left|Q_{k}(x)\right|\right] \\
& \geq \sup _{x \in \mathbb{T}}\left[\left|\left(\pi_{\Lambda_{m}} P\right)(x)\right|+\left|P(x)-\left(\pi_{\Lambda_{m}} P\right)(x)\right|\right]
\end{aligned}
$$

Although it is possible that $\mathcal{C}_{\Lambda}(\mathbb{T})$ has (C-UMAP) when $\Lambda$ is a Hilbert set, we have, however,

Proposition 11. If $\mathcal{C}_{\Lambda}(\mathbb{T})$ has (UMAP), then $\Lambda$ cannot contain any IP-set.

Let us recall that an IP-set is the set

$$
\left\{\sum_{j=1}^{n} \varepsilon_{j} p_{j}: \varepsilon_{j}=0 \text { or } 1, n \geq 1\right\}
$$

of all the finite sums of a given sequence $\left(p_{n}\right)_{n \geq 1}$ in $\mathbb{N}^{*}$.

Proof. This follows from the lemma below, by choosing $k_{n}=\sum_{j=1}^{n} p_{2 j}$ and $l_{n}=\sum_{j=1}^{n} p_{2 j+1}$.

LEMMA 12. If there are two sequences $\left(k_{n}\right)_{n \geq 1}$ and $\left(l_{n}\right)_{n \geq 1}$ in $\mathbb{Z}$ such that

$$
\left|k_{n}\right|,\left|l_{n}\right|,\left|k_{n}+l_{n}\right| \underset{n \rightarrow \infty}{\longrightarrow} \infty
$$

and such that $\Lambda \supseteq\left\{0, k_{n}, l_{n}, k_{n}+l_{n}: n \geq 1\right\}$, then $\mathcal{C}_{\Lambda}(\mathbb{T})$ cannot have (UMAP).

Proof. Assume that $\mathcal{C}_{\Lambda}(\mathbb{T})$ has (UMAP). From Lemma 5 , there is a sequence $\left(A_{n}\right)_{n \geq 1}$ of trigonometric polynomials such that

$$
\begin{gathered}
\forall f \in \mathcal{C}_{\Lambda} \quad\left\|A_{n} * f-f\right\|_{\infty} \underset{n \rightarrow \infty}{\longrightarrow} 0, \\
\left\|\operatorname{Id}-2 C_{A_{n}}\right\|=1+\varepsilon_{n}, \quad \text { where } \varepsilon_{n} \underset{n \rightarrow \infty}{\longrightarrow} 0,
\end{gathered}
$$

and where $C_{A_{n}}$ is the convolution operator associated with $A_{n}$.

We choose $u(x) \equiv-1$ and $n_{0}$ large enough to have $\left\|A_{n_{0}} * u-u\right\|_{\infty} \leq 1 / 2$ and $\varepsilon_{n_{0}}<1 / 33$.
We also choose $n$ large enough in order that $k_{n}, l_{n}, k_{n}+l_{n} \notin \operatorname{spec}\left(A_{n_{0}}\right)$. Then the functions $v$ and $w$ defined by

$$
v(x)=e^{i k_{n} x} \text { and } w(x)=e^{i l_{n} x}\left(1+e^{i k_{n} x}\right)
$$

are in $\mathcal{C}_{\Lambda}$ and satisfy $v * A_{n_{0}}=0$ and $w * A_{n_{0}}=0$. We then have

$$
\begin{aligned}
\|u+v+w\|_{\infty} & =\sup _{x \in \mathbb{T}}|u(x)+v(x)+w(x)| \leq \sup _{x \in \mathbb{T}}(|u(x)+v(x)|+|w(x)|) \\
& =\sup _{x \in \mathbb{T}}\left(\left|-1+e^{i k_{n} x}\right|+\left|1+e^{i k_{n} x}\right|\right)=2 \sqrt{2} .
\end{aligned}
$$

On the other hand, since $A_{n_{0}} *(v+w)=0$, we have

$$
\begin{aligned}
\|\left(\operatorname{Id}-2 C_{A_{n_{0}}}\right)(u+v+w) & \left\|_{\infty}=\right\| v+w+\left[u-2\left(A_{n_{0}} * u\right)\right] \|_{\infty} \\
& \geq\|v+w-u\|_{\infty}-2\left\|u-A_{n_{0}} * u\right\|_{\infty} \\
& \geq \sup _{x \in \mathbb{T}}|v(x)+w(x)-u(x)|-1 \\
& =\sup _{x \in \mathbb{T}}\left(\left|e^{i k_{n} x}+e^{i l_{n} x}\left(1+e^{i k_{n} x}\right)+1\right|\right)-1=3 .
\end{aligned}
$$

We must then have $3 \leq\left(1+\varepsilon_{n_{0}}\right) 2 \sqrt{2}$, which is not possible since $\varepsilon_{n_{0}}<$ $1 / 33$.

Remark. Actually, the proof gives $\liminf \varepsilon_{n} \geq \sqrt{2}-1$.

As a consequence of Lemma 12, we have:

Corollary 13. If $\Lambda \supseteq \Lambda_{1}+\Lambda_{2}$ for two infinite sets $\Lambda_{1}, \Lambda_{2} \subseteq \mathbb{N}$, then $\mathcal{C}_{\Lambda}(\mathbb{T})$ does not have (UMAP).

Indeed, by translation, we may assume that $0 \in \Lambda_{1}$ and $0 \in \Lambda_{2}$.

Remark. Y. Meyer showed ([18], Th. 3a, p. 558) that $\Lambda=\left\{n_{k}+n_{l}\right.$ : $k, l \geq 1\}$ is a $\Lambda(p)$-set for all $p \geq 1$ if $\left(n_{k}\right)_{k \geq 1}$ is a Hadamard sequence. However, $\mathcal{C}_{\Lambda}(\mathbb{T})$ does not have (UMAP) by Corollary 13.

Moreover, S. Neuwirth pointed out to me that Lemma 12 shows that the same holds for the Sidon set $\Lambda=\{0\} \cup\left\{2^{n}: n \in \mathbb{N}\right\}$. Hence (UMAP) seems to be connected to the rapidity of the growth to infinity.

Faving ( $\mathbb{C}-$ UMAP) is a strong hypothesis on a Banach space, and we might expect that $\Lambda$ has null uniform density whenever $\mathcal{C}_{\Lambda}(T)$ has $\left(\mathbb{C}\right.$-UMAP). Indeed, this density is null when $\mathcal{C}_{\Lambda}(\mathbb{T})$ has no subspace isomorphic to $c_{0}([17]$, Th. 3 ), or merely when $\Lambda$ contains no Hilbert subset ([15], Cor. 8). We have not been able to solve this question, but Lemma 12 provides a partial answer.

Let us recall the definition of the uniform density of a subset $\Lambda \subseteq \mathbb{Z}$ :

$$
d^{*}(\Lambda)=\lim _{h \rightarrow \infty}\left[\sup _{a \in \mathbb{Z}} \frac{\operatorname{card}(\Lambda \cap] a, a+h])}{h}\right] .
$$


PropostTION 14. $d^{*}(\Lambda) \leq 1 / 2$ as soon as $\mathcal{C}_{\Lambda}(\mathbb{T})$ has (UMAP).

Proof. Let $\Lambda$ be such that $d^{*}(\Lambda)>1 / 2$. We may suppose that $0 \in \Lambda$.

Then the proposition is a consequence of Lemma 12 and of the following result of N. Hindman ([12], Th. 3.4): if $d^{*}(\Lambda)>1 / 2$, then for each $n \in \mathbb{N}$ we have $d^{*}(\Lambda \cap(\Lambda-n)) \geq 2 d^{*}(\Lambda)-1$. We should note, however, that the definition of $d^{*}(\Lambda)$ given by $N$. Hindman is (at least formally) slightly different from ours, and he assumes that $\Lambda \subseteq \mathbb{N}$, but his proof works as well in our setting.

Indeed, choosing $k_{1} \in \Lambda$ with $k_{1} \neq 0$, we have, by Findman's result, $d^{*}\left(\Lambda \cap\left(\Lambda-k_{1}\right)\right)=d^{*}\left(\Lambda \cap\left(\Lambda-\left|k_{1}\right|\right)\right)>0$, and so we can find an $l_{1} \in \Lambda \cap\left(\Lambda-k_{1}\right)$ with $\left|l_{1}\right| \geq\left|k_{1}\right|+1 ;$ then $k_{1}, l_{1}, k_{1}+l_{1} \in \Lambda$. We iterate the same process with $\Lambda_{n}=\Lambda \backslash[-n,+n]$ instead of $\Lambda$; we still have $d^{*}\left(\Lambda_{n}\right)>1 / 2$ and we choose $k_{n} \in \Lambda_{n}$ and find $l_{n} \in \Lambda_{n} \cap\left(\Lambda_{n}-k_{n}\right)$ such that $\left|l_{n}\right| \geq\left|k_{n}\right|+n$.

Our last result is the existence of a linear invariant lifting for $L_{\Lambda}^{\infty}$.

PRopostTION 15. If $\mathcal{C}_{\Lambda}(G)$ has (UMAP), then there exixts a linear in variant lifting $\mathcal{R}: L_{\Lambda}^{\infty} \rightarrow \mathrm{Ba}(G)$, where $\mathrm{Ba}(G)$ is the space of first Baire class functions. In particular, $\Lambda$ has the Godefroy-Lust-Piquard's property (e) (see [9], Def. V.1).

Proof. Let $\left(A_{n}\right)_{n \geq 1}$ be a sequence of trigonometric polynomials such that

$$
\begin{aligned}
& \forall f \in \mathcal{C}_{A} \quad\left\|A_{n} * f-f\right\|_{\infty} \underset{n \rightarrow \infty}{\longrightarrow} 0, \\
& \left\|\operatorname{Id}-2 C_{A_{n}}\right\|=1+\varepsilon_{n}, \quad \text { where } \quad \varepsilon_{n} \underset{n \rightarrow \infty}{\longrightarrow} 0,
\end{aligned}
$$

and where $C_{A_{n}}$ is the convolution operator associated with $A_{n}$

For all $\Phi \in \mathcal{C}_{A}^{* *}$, the limit

$$
T(\Phi)=\underset{n \rightarrow \infty}{w^{*}-\lim _{n}} C_{A_{n}}^{* *}(\Phi)
$$

exists (see [5], proof of Th. IV.1, or from [3], Th. 3.8, we may suppose that the series $\sum\left(C_{A_{n+1}-A_{n}}\right)$ is weakly unconditionally convergent). Hence, for every $\mu \in \mathcal{M}$ (the space of Radon measures on $G$ ), we have $\langle T(\Phi), \mu\rangle=$ $\lim _{n \rightarrow \infty}\left\langle\Phi, C_{A_{n}}^{*}(\mu)\right\rangle$. But $C_{A}^{*}(\mu)=\breve{A}_{n} * \mu \in L^{1}$ and so the above limit depends only on $\Phi_{\mid L^{1}}$ which is an element of $L_{\Lambda}^{\infty}$. Since every element of $L_{\Lambda}^{\infty}$ can be written in this way, we can then define for every $g \in L_{\Lambda}^{\infty}$ an element $\widetilde{T}(g) \in \mathcal{C}_{\Lambda}^{* *}$ which satisfies, for every $\mu \in \mathcal{M}$,

$$
\langle\widetilde{T}(g), \mu\rangle=\lim _{n \rightarrow \infty}\left\langle g, \check{A}_{n} * \mu\right\rangle=\lim _{n \rightarrow \infty}\left\langle A_{n} * g, \mu\right\rangle .
$$

In particular, we can define, for every $x \in G$,

$$
(\mathcal{R} g)(x)=\lim _{n \rightarrow \infty}\left\langle A_{n} * g, \delta_{x}\right\rangle
$$

which defines an element of the first Baire class since $A_{n} * g$ is a continuous function.

Moreover, by dominated convergence, for every $\varphi \in L^{1}$ we have

$$
\begin{aligned}
\int_{G}(\mathcal{R} g)(x) \varphi(x) d x & =\lim _{n \rightarrow \infty} \int_{G}\left(A_{n} * g\right)(x) \varphi(x) d x \\
& =\lim _{n \rightarrow \infty}\left(A_{n} * g * \check{\varphi}\right)(0)=(g * \check{\varphi})(0)=\langle g, \varphi\rangle
\end{aligned}
$$

since $g * \check{\varphi} \in \mathcal{C}_{\Lambda}$. Hence $\mathcal{R} g$ is a representative of $g$.

Now, the linear form $\varrho: L_{\Lambda}^{\infty} \rightarrow \mathbb{C}$ defined by $\varrho(g)=(\mathcal{R} g)(0)$ is of the first Baire class in the $w^{*}$-topology of $L_{\Lambda}^{\infty}$ and $\varrho(f)=f(0)$ for every $f \in \mathcal{C}_{\Lambda}$ so that $\Lambda$ has Godefroy-Lust-Piquard's property $(\varrho)$

As a corollary of Theorem 10 and Proposition 15, we have

THEOREM 16. There is a set $\Lambda \subseteq \mathbb{Z}$ which has Godefroy-Lust-Piquard's lifting property $(\varrho)$, but which is not a Rosenthal set.

This answers negatively a question of [9], where it was conjectured that the only possibility to have property $(\varrho)$ was that all the elements of $L_{\Lambda}^{\infty}$ were already continuous.

R e mark. As recalled at the beginning, there is no Sidon set with constant 1 in $\mathbb{Z}$. However, it is not known if the (C-UMAP) can be realized with an approximating sequence $\left(R_{n}\right)_{n \geq 1}$ such that $\left\|\mathrm{Id}-(1+\lambda) R_{n}\right\|=1$. Nevertheless, there is no increasing sequence of finite subsets $F_{n} \subseteq \mathbb{Z}$ such that $\bigcup_{n>1} F_{n}=\Lambda$ and $\left\|\operatorname{Id}-(1+\lambda) \pi_{F_{n}}\right\|=1$ for all $\lambda$ with $|\lambda|=1$.

Indeed, if such a sequence existed, we could pick $n_{1} \in F_{1}$ and $n_{2} \notin F_{1}$, and then $F_{n_{2}}$ such that $n_{2} \in F_{n_{2}}$, and finally $n_{3} \notin F_{1} \cup F_{n_{2}}$. Then we would have

$$
\begin{aligned}
\left\|a_{1} e_{n_{1}}+a_{2} e_{n_{2}}+a_{3} e_{n_{3}}\right\|_{\infty} \\
\geq \sup _{|\lambda|=1}\left\|\left(\operatorname{Id}-(1+\lambda) \pi_{F_{1}}\right)\left(a_{1} e_{n_{1}}+a_{2} e_{n_{2}}+a_{3} e_{n_{3}}\right)\right\|_{\infty} \\
=\sup _{|\lambda|=1}\left\|-\lambda a_{1} e_{n_{1}}+a_{2} e_{n_{2}}+a_{3} e_{n_{3}}\right\|_{\infty} \\
=\left|a_{1}\right|+\left\|a_{2} e_{n_{2}}+a_{3} e_{n_{3}}\right\|_{\infty} \\
\geq\left|a_{1}\right|+\sup _{|\lambda|=1}\left\|\left(\operatorname{Id}-(1+\lambda) \pi_{F_{n_{2}}}\right)\left(a_{2} e_{n_{2}}+a_{3} e_{n_{3}}\right)\right\|_{\infty} \\
=\left|a_{1}\right|+\sup _{|\lambda|=1}\left\|-\lambda a_{2} e_{n_{2}}+a_{3} e_{n_{3}}\right\|_{\infty}=\left|a_{1}\right|+\left|a_{2}\right|+\left|a_{3}\right| .
\end{aligned}
$$

Therefore, we would conclude that $\left\{n_{1}, n_{2}, n_{3}\right\}$ is a Sidon set with constant 1 , which is not possible. 
For the sake of completeness, we now sketch a proof of this last fact, different from that of [2], which was given to us by G. Pisier. We may assume that $n_{1}=0$ and write $n_{2}=k, n_{3}=l$. Let $\theta_{j}(j=1,2)$ be the character of $\mathbb{T}^{2}$ which associates with $\left(x_{1}, x_{2}\right)$ the value $e^{i x_{j}}$. If $\{0, k, l\}$ where a Sidon set with constant 1 , then for every $a, b \in \mathbb{C}$ we would have $\left\|1+a e_{k}+b e_{l}\right\|_{\infty}=$ $\left\|1+a \theta_{1}+b \theta_{2}\right\|_{\infty}$, and then also $\left\|1+a e_{k}+b e_{l}\right\|_{p}=\left\|1+a \theta_{1}+b \theta_{2}\right\|_{p}$ for every $p \in\left[1, \infty\left[\right.\right.$ (see [23], or [20], Th. 1). But then, from ([26], Th. I), $\left(e_{k}, e_{l}\right)$ and $\left(\theta_{1}, \theta_{2}\right)$ would have the same distribution, and this is false.

Added in proof. $S$. Neuwirth showed that $\mathcal{C}_{\Lambda}(\mathbb{T})$ does not have (C-UMAP) for $\Lambda=$ $\left\{q^{n}: n \geq 1\right\}$, and $q \geq 2$ an integer, but $\mathcal{C}_{A}(\mathbb{T})$ does have (C-UMAP) if $\Lambda=\left\{n_{k}: k \geq 1\right\}$ with $n_{k+1} / n_{k} \rightarrow \infty$ as $k \rightarrow \infty$.

\section{References}

[1] G. F. Bachelis and S. E. Ebenstein, On $\Lambda(p)$ sets, Pacific J. Math. 54 (1974), 35-38.

[2] D. L. Cartwright, R. B. Howlett and J. R. McMullen, Extreme values for the Sidon constant, Proc. Amer. Math. Soc. 81 (1981), 531-537.

[3] P. Casazza and N. J. Kalton, Notes on approximation properties in separable Banach spaces, in: Geometry of Banach Spaces, P. F. X. Müller and W. Schachermayer (eds)., London Math. Soc. Lecture Note Ser. 158, Cambridge Univ, Press, $1990,49-63$.

[4] G. Godefroy, On Riesz subsets of abelian discrete groups, Israel J. Math. 61 (1988), 301-331.

[5] G. Godefroy and N. J. Kalton, Commuting approximation properties, preprint.

[6] G. Godefroy, N. J. Kalton and D. Li, On subspaces of $L^{1}$ which embed into $\ell_{1}$ J. Reine Angew. Math. 471 (1996), 43-75.

[7] G. Godefroy, N. J. Kalton and P. D. Saphar, Unconditional ideals in Banach spaces, Studia Math. 104 (1993), 13-59.

[8] G. Godefroy and D. Li, Some natural families of M-ideals, Math. Scand. 66 (1990) 249-263.

[9] G. Godefroy and F. Lust-Piquard, Some applications of geometry of Banach spaces to harmonic analysis, Colloq. Math. 60/61 (1990), 443-456.

[10] G. H. Hardy and E. M. Wright, An Introduction to the Theory of Numbers, 4th ed., Oxford Univ. Press, 1960.

[11] S. Hartman, Some problems and remarks on relative multipliers, Colloq. Matt]. 54 (1987), 103-111.

[12] N. Hindman, On density, translates, and pairwise sums of integers, J. Combin. Theory Ser. A 33 (1982), 147-157.

[13] M. I. Kadec and A. Pełczyński, Bases, lacunary sequences and complemented subspaces in the spaces $L_{p}$, Studia Math. 21 (1.962), 161 -176.

[14] N. J. Kalton, Spaces of compact operators, Math. Ann. 208 (1974), 267-278.

[15] D. Li, On Hilbert sets and $C_{\Lambda}(G)$-spaces with no subspace isomorphic to $c_{0}$, Colloc. Math. 63 (1.995), 67-77.

[16] F. Lust-Piquard, Ensembles de Rosenthal et ensembles de Riesz, C. R. Acad. Sci. Paris Sér. A $282(1976), 833-835$

[17] F. Lust-Piquard, Eléments ergodiques et totalement ergodiques dans $L^{\infty}(\Gamma)$, Studia Math. 69 (1981), 191-225.

[18] Y. Meyer, Endomorphismes des idéaux fermés de $L^{1}(G)$, classes de Hardy et séries de Fourier lacunaires, Ann. Sci. Ecole Norm. Sup. (4) 1 (1968), 499-580.

[19] -, Algebraic Numbers and Harmonic Analysis, North-Holland, 1972

[20] A. Petczyński, On commensurate sequences of characters, Proc. Amer. Math Soc. 104 (1988), 525-531.

[21] A. Pełczyński and P. Wojtaszczyk, Banach spaces with finite dimensional expansions of identity and universal bases of finite dimensional subspaces, Studia Math. 40 (1971), 91-108.

[22] G. Pisier, Bases, suites lacunaires dans les espaces $L^{p}$ d'après Kadec et Pełczyński, Sém. Maurey-Schwartz, exposé 18, Ecole Polytechnique, Paris, 1973.

[23] -, Les inégalités de Khintchine-Kahane d'après C. Borell, Sém. Géométrie des Espaces de Banach 1977-1978, exposé VII, Ecole Polytechnique, Paris.

[24] H. P. Rosenthal, On trigonometric series associated with weak ${ }^{*}$ closed subspaces of continuous functions, J. Math. Mech. 17 (1967), 485-490.

[25] W. Rudin, Trigonometric series with gaps, ibid. 9 (1960), 203-227.

[26],$- L^{p}$-isometries and equimeasurability, Indiana Univ. Math. J. 25 (1976), 215-228.

Analyse Harmonique

Université Paris-Sud

Mathématiques

Bâtiment 425

91405 Orsay, France
Equipe d'Analyse

Université Paris VI 4 Place Jussieu 75252 Paris Cedex 05, France E-mail: daniel.li@math.u-psud.f 